\title{
Common Allergens Identified Based on Patch Test Results in Patients with Suspected Contact Dermatitis of the Scalp
}

\author{
Nouf M. Aleid ${ }^{a}$ Raymond Fertig ${ }^{b}$ Austin Maddy $^{b} \quad$ Antonella Tosti $^{b}$ \\ ${ }^{a}$ Department of Dermatology, Prince Sultan Military Medical City, Riyadh, Saudi Arabia; ${ }^{b}$ Department of \\ Dermatology and Cutaneous Surgery, Miller School of Medicine, University of Miami, Miami, FL, USA
}

\section{Keywords}

Nickel · Cobalt · Carba mix · Fragrances · Balsam of Peru ·

Propylene glycol · Androgenetic alopecia · Telogen

effluvium · Seborrheic dermatitis

\begin{abstract}
Background: Contact dermatitis of the scalp is common and might be caused by many chemicals including metals, ingredients of shampoos and conditioners, dyes, or other hair treatments. Eliciting a careful history and patch tests are necessary to identify the responsible allergen and prevent relapses. Objectives: To identify allergens that may cause contact dermatitis of the scalp by reviewing patch test results. Methods: We reviewed the records of 1,015 patients referred for patch testing at the Dermatology Department of the University of Miami. A total of 226 patients ( 205 females and 21 males) with suspected scalp contact dermatitis were identified, and the patch test results and clinical data for those patients were analyzed. Most patients were referred for patch testing from a specialized hair clinic at our institution. $\boldsymbol{R e}$ sults: The most common allergens in our study population were nickel (23.8\%), cobalt (21.0\%), balsam of Peru (18.2\%), fragrance mix (14.4\%), carba mix (11.6\%), and propylene glycol (PG) (8.8\%). The majority of patients were females aged
\end{abstract}

\section{KARGER}

(c) 2016 S. Karger AG, Basel

E-Mail karger@karger.com

www.karger.com/sad
40-59 years, and scalp itching or burning were reported as the most common symptom. Conclusion: Frequent sources of allergens for metals include hair clasps, pins, and brushes, while frequent sources of allergens for preservatives, fragrance mix, and balsam of Peru include shampoos, conditioners, and hair gels. Frequent sources of allergens for PG include topical medications.

(c) 2016 S. Karger AG, Basel

\section{Introduction}

Contact dermatitis of the scalp is not as common as contact dermatitis of other areas of the body. This is probably due to the anatomical features of the scalp skin, which is thick and rich with pilosebaceous units [1]. $\mathrm{Pa}$ tients with scalp contact dermatitis often do not have evident skin lesions on the scalp, but instead present with acute or chronic scalp itch or hair loss [2]. Typical eczematous lesions may be seen at the hairlines, on the ears, at the retroauricular region, and on the neck.

Nouf M. Aleid and Raymond Fertig are both first authors.
Raymond Fertig

Department of Dermatology and Cutaneous Surgery Miller School of Medicine, University of Miami

1475 NW 12th Ave., 2nd Floor, Miami, FL 33136 (USA)

E-Mail raymondfertig@gmail.com 
Table 1. Most common allergens identified in suspected cases of scalp contact dermatitis

\begin{tabular}{|c|c|c|c|c|}
\hline & Allergen & Patients & $\%$ & Source relevant to the scalp ${ }^{1}$ \\
\hline \multirow[t]{2}{*}{ Metals } & nickel & 43 & 23.8 & \multirow{2}{*}{$\begin{array}{l}\text { hair clasps, metal head pins } \\
\text { metal hair pins, hair dyes (light brown) } \\
\text { textile dye fixing agents, leather }\end{array}$} \\
\hline & cobalt & 38 & 21.0 & \\
\hline \multirow[t]{2}{*}{ Fragrances } & balsam of Peru & 33 & 18.2 & \multirow{2}{*}{$\begin{array}{l}\text { shampoos, conditioners, hair tonics or pomades } \\
\text { shampoos, hair conditioners, tonics, oils, hair gels }\end{array}$} \\
\hline & fragrance mix & 26 & 14.4 & \\
\hline Vehicles & propylene glycol & 16 & 8.8 & hair products (shampoos, conditioners, gels, colors, minoxidil) \\
\hline Hair products & ammonium persulfate & 13 & 7.2 & oxidizers in hair bleaches \\
\hline Preservatives & Kathon CG & 9 & 5.0 & $\begin{array}{l}\text { preservatives, shampoos, hair conditioners, detangler hair } \\
\text { styling mousses, foams, gels, hair colors } \\
\text { preservatives, shampoos, hair conditioners, hair styling } \\
\text { products, hair color, dyes, permanent wave solutions } \\
\text { preservatives in vaccines, not relevant } \\
\text { preservatives, shampoos, hair conditioners, tonics, spray hair } \\
\text { styling gels, mousses }\end{array}$ \\
\hline Hair coloring & PPD & 6 & 3.3 & $\begin{array}{l}\text { permanent hair dye products, henna tattoos, textile or fur } \\
\text { dyes, black rubber }\end{array}$ \\
\hline \multirow[t]{3}{*}{ Topical medications } & bacitracin & 4 & 2.1 & \multirow{3}{*}{$\begin{array}{l}\text { not relevant } \\
\text { not relevant }\end{array}$} \\
\hline & neomycin & 5 & 2.8 & \\
\hline & minoxidil $^{2}$ & 4 & 5.5 & \\
\hline \multirow[t]{2}{*}{ Others } & disperse blue dye & 7 & 3.9 & \multirow{2}{*}{$\begin{array}{l}\text { cross-sensitivity to PPD, which is present in most hair dyes } \\
\text { hair care products and perfumes }\end{array}$} \\
\hline & anise alcohol & 5 & 2.8 & \\
\hline
\end{tabular}

Kathon CG, methylchloroisothiazolinone/methylisothiazolinones; PPD, paraphenylenediamine.

${ }^{1}$ Data from the American Contact Dermatitis Society (ACDS) (http://www.contactderm.org), with modification. ${ }^{2}$ Minoxidil was tested in only 73 patients and not in all subjects, of whom 4 tested positive.

Patients may attribute their symptoms to the use of a new shampoo or hair dye, various cosmetic products, keratin treatments, or other hair treatments. Therefore, eliciting a careful history is important since the scalp is exposed to many substances. Furthermore, patients may have concomitant preexisting hair disorders that cause scalp irritation. Contact scalp dermatitis can present in the healthy scalp or in association with a preexisting scalp condition.

\section{Materials and Methods}

The medical records of 1,015 patients referred for patch testing to the Dermatology Department of the University of Miami were reviewed retrospectively and independently, as approved by the University of Miami Institutional Review Board. Patients were seen in an outpatient dermatology clinic for contact dermatitis from January 1, 2012 to March 30, 2016. A dermatology fel- low sorted the cases and identified 226 patients with suspected scalp contact dermatitis. The patch test results and clinical data for those patients were collected and analyzed. Data analysis was conducted from April 1 to May 30, 2016. Data collected from patient records included age, gender, preexisting scalp dermatoses, patch test results, and anatomical site of areas affected that showed evidence of dermatitis in addition to the scalp. Data were entered using Microsoft Excel 2007. Subsequently, 187 of the 226 patients were referred to the patch test clinic of a clinic specialized in hair and scalp disorders from the same institution. Reasons for patch test referral included clinical or dermoscopic signs suggestive of possible contact dermatitis, such as scalp erythema, scaling, or excruciations, and symptoms such as scalp itching or scalp burning.

The series chosen for the patch test (Chemotechnique Diagnostics, Vellinge, Sweden) was selected based on patient history. This included standard, cosmetic, fragrances, hair series, and patient's personal products, with rinse-off products tested as open patch tests. Patch tests and readings were performed by the same physician using the International Contact Dermatitis Research Group guidelines [3]. 
Table 2. Demographics of total suspected scalp contact dermatitis patients

\begin{tabular}{lrllrrr}
\hline $\begin{array}{l}\text { Age } \\
\text { categories }\end{array}$ & Females $\%$ & Males & \multicolumn{1}{l}{ Total } & \multicolumn{1}{l}{$\%$} \\
\hline $0-19$ & 4 & 1.95 & 1 & 4.8 & 5 & 2.2 \\
$20-39$ & 59 & 28.8 & 6 & 28.5 & 65 & 28.8 \\
$40-59$ & 82 & 40.0 & 5 & 23.8 & 87 & 38.5 \\
$60-79$ & 56 & 27.3 & 7 & 33.3 & 63 & 27.9 \\
$80-100$ & 4 & 1.95 & 2 & 9.5 & 6 & 2.7 \\
\hline Total & 205 & 100 & 21 & 100 & 226 & 100 \\
\hline
\end{tabular}

Table 3. Symptoms experienced in suspected scalp contact dermatitis patients

\begin{tabular}{lcr}
\hline Symptoms & Patients & $\%$ \\
\hline Itching/burning & 194 & 85.8 \\
Hair thinning/shedding & 133 & 58.8 \\
Hair thinning/shedding and itching/burning) & 115 & 50.9 \\
Pain & 6 & 2.7 \\
\hline
\end{tabular}

\section{Results}

Data from 226 patients (205 females and 21 males) referred for patch testing due to possible allergic contact dermatitis of the scalp were reviewed. The allergens most frequently eliciting a positive reaction in the patch tests are reported in Table 1. In total, 181 positive allergen reactions were recorded from the total sample population of 226 patients $(80.1 \%)$. In 45 patients no allergen could be identified. The most common allergens in our study population were nickel (23.8\%), cobalt (21.0\%), balsam of Peru (18.2\%), fragrance mix (14.4\%), carba mix (11.6\%), and propylene glycol (PG) (8.8\%). Other less frequent allergens included minoxidil, anise alcohol, quaternium-15 and paraphenylenediamine (PPD). The relevance of patch test reactions was established after reviewing the patients' habits and specific products used. Female patients were heavily represented, with the most common age range being $40-59$ years (Table 2 ).

\section{Affected Areas and Symptoms}

Scalp erythema was reported in 177 cases (78.3\%). In 92 patients, other body areas were affected, including the face $(16.4 \%)$, the trunk $(11.0 \%)$, the extremities $(8.4 \%)$,
Table 4. Primary scalp condition in suspected scalp contact dermatitis patients

\begin{tabular}{lll}
\hline Primary scalp conditions & Patients & $\%$ \\
\hline Androgenetic alopecia & 78 & 34.5 \\
Normal scalp & 44 & 19.5 \\
Telogen effluvium & 22 & 9.7 \\
Chronic telogen effluvium & 19 & 8.4 \\
Seborrheic dermatitis & 14 & 6.2 \\
Alopecia areata incognito & 11 & 4.9 \\
Psoriasis & 9 & 4.0 \\
Scaling of scalp/dandruff & 9 & 4.0 \\
Lichen planopilaris & 8 & 3.5 \\
Alopecia areata & 6 & 2.7 \\
Psoriasiform dermatitis of the scalp & 6 & 2.7 \\
Atopic dermatitis & 4 & 1.8 \\
Tractional alopecia & 4 & 1.8 \\
Hair weathering/breakage & 3 & 1.3 \\
Trichodynia & 3 & 1.3 \\
Central centrifugal cicatricial alopecia & 2 & 0.9 \\
Frictional alopecia & 2 & 0.9 \\
Frontal fibrosing alopecia & 2 & 0.9 \\
Lichen simplex chronicus & 2 & 0.9 \\
Sebopsoriasis & 2 & 0.9 \\
Trichorrhexis nodosa & 2 & 0.9 \\
Mixed connective tissue disease & 2 & 0.9 \\
\hline
\end{tabular}

and the neck (4.9\%). The most commonly reported symptoms were scalp itching and burning (194 cases, 85.8\%). Hair thinning and shedding was a common complaint as most patients had consulted the hair clinic because of hair loss (58.8\%) (Table 3).

\section{Primary Scalp Conditions}

The majority of patients $(187,82.7 \%)$ suffered from a preexisting scalp condition, while 44 (19.5\%) had no primary disease of the scalp (Table 4 ). The most common preexisting scalp dermatoses were androgenetic alopecia (34.5\%), acute telogen effluvium (9.7\%), chronic telogen effluvium (8.4\%), seborrheic dermatitis (6.2\%), and alopecia areata $(4.9 \%)$. Associated scalp disorders included psoriasis, psoriasiform dermatitis of the scalp, lichen planopilaris, alopecia areata, atopic dermatitis, and frontal fibrosing alopecia, among others.

\section{Discussion}

Based on the patch test results, our study identified metals (including nickel and cobalt) as the most common allergens. In addition to metals, the other common aller- 
gens identified included balsam of Peru, fragrance mix, carba mix, PG, ammonium persulfate (APS), preservatives, PPD, and minoxidil. Frequent causes of contact dermatitis of the scalp in our patient population included brushes and combs, hair rollers, hair clasps and pins, hair dyes, shampoos, conditioners, hair gels or oils, as well as leave-on hair products.

The study conducted by Hillen et al. [4] illustrates the spectrum of allergens in scalp dermatitis. This multicenter study analyzed data over a 10-year span from 1993 to 2003 covering over 40 dermatology clinics from the Information Network of Departments of Dermatology (IVDK). Data from 1,320 patients who underwent patch testing to confirm or rule out allergic contact dermatitis of the scalp were analyzed. Hair dyes such as p-aminoazobenzene, PPD, and toluene-2,5-diamine were the most prevalent allergens in their patient population. Medical products, hair dyes and bleaches, and hair cleansing products together caused nearly two-thirds of the positive patch test reactions. This study concluded that testing of patients' own products is an important part of the diagnostic workup for contact scalp dermatitis, as some ingredients of hair dyes are not contained in the patch test series.

\section{Nickel}

Nickel is a ubiquitous metal used in a wide variety of products and is the most common allergen encountered worldwide [5]. Of the 181 patients in whom an allergen was identified as causing scalp contact dermatitis, 43 (23.8\%) showed an allergic response to nickel, making it the most prevalent of the allergens identified in this study. These results are similar to the observations made in the study by Hillen et al. [4], which recorded a positive nickel reaction in $15.5 \%$ of patients. Scalp exposure to nickel can be due to combs, brushes, rollers, hair clasps, and metal hairpins. Nickel allergy was judged to be relevant in 15 patients who affirmed the use of metal tools for hair grooming and styling. Several of our patients used metal bristle brushes, which are very popular for hair styling. Additionally, other metal tools associated with scalp dermatitis and itching in our patients were metal hair clasps. Hair clasps might release high levels of nickel and have been reported as a possible cause of scalp dermatitis in the past $[6,7]$. A study from Denmark showed that $19.3 \%$ of adult hair clasps and $79.4 \%$ of child hair clasps had excessive nickel release (i.e., $>0.5 \mu \mathrm{g}$ nick$\mathrm{el} / \mathrm{cm}^{2} /$ week) using the dimethylglyoxime test [8]. Nickel exposure may not always be obvious, as shown by recent reports describing nickel scalp dermatitis caused by head pins used in a neurosurgical operation [9] and by headbands containing nickel [10].

\section{Cobalt}

Cobalt is a metal found in nature. It is commonly used with nickel for metal plating, and added to alloys to make more robust tools and parts. A positive test to cobalt was seen in 38 patients $(21.0 \%)$. Of note, 28 of these 38 patients (73.7\%) also tested positive to nickel. This finding is much higher than results from the study conducted by Hillen et al. [4], which recorded cobalt allergy in only $5.6 \%$ of patients. Approximately $80 \%$ of individuals with cobalt sensitivity have co-sensitivity to other metals, with the predominant co-sensitivity being nickel [11]. One study has shown that patients with cobalt and nickel coreactions were more likely to be female (OR 3.1, 95\% CI $1.5-6.8, p=0.0038$ ) and more likely to have scalp dermatitis (OR 3.9, 95\% CI 1.3-11.7, $p=0.0286$ ) compared to those who did not react to metals [12]. Additionally, those who reacted to cobalt had increased odds of scalp dermatitis [12]. It has been postulated that nickel sensitization and preexisting dermatitis are often prerequisites for cobalt sensitization [13].

Information about the presence of cobalt in products is often not apparent. Thus, establishing the relevant source of cobalt responsible for the scalp dermatitis reaction can be difficult. However, cobalt is present in hair dyes [14], particularly light brown hair dyes, and a large contingent of our population (65 patients, $35.9 \%$ ) regularly dyed their hair. Therefore, we suspect that hair dyes containing cobalt may have been responsible for at least some of the 38 positive patch test results to cobalt, although we could not confirm the presence of cobalt in our patients' hair dye products. In addition to hair dyes, cobalt may also be found in costume jewelry, detergents, antiperspirants, solid soaps, and cosmetics [14].

\section{Fragrances}

The second most common allergen identified in our series was fragrances. Of the 181 patients in our study population, $59(32.6 \%)$ had a positive patch test to either balsam of Peru (Myroxylon pereirae) (33, 18.2\%), fragrance mix I $(22,12.2 \%)$, or fragrance mix II $(4,2.2 \%)$. All of these reactions were judged to be relevant as it was confirmed that these particular subjects were utilizing shampoos, conditioners, and other styling products that contained fragrances.

Balsam of Peru is the aromatic sap derived from the tree Myroxylon balsamum that grows in Central and South America. Balsam of Peru is a complex substance
Aleid/Fertig/Maddy/Tosti 
that contains many potential allergens such as benzoic acid, benzyl acetate, benzyl benzoate, and cinnamic acid, among others. It is a sweet-smelling natural substance that is commonly found in fragrances. Patients should be suspicious of aromatherapy products, scented oils, scented candles, air fresheners, deodorizers, or incense with a cinnamon, vanilla, or clove aroma. It is wise to choose only fragrance-free products because products labeled as "unscented" may actually contain a masking fragrance. Balsam of Peru can be found in hair tonic products, pomade, shampoos, conditioners, shaving lotion, aftershave perfume, cologne, and cosmetic products with fragrance.

In addition to its use in fragrances, balsam of Peru can also be found in foods, drinks, and medicines. In a study by Salam and Fowler [15], 1 patient had significant improvement of scalp dermatitis by following a strict diet that excluded balsam of Peru. In this study, the most common balsam-related foods deemed responsible for causing dermatitis included tomatoes, citrus, and spices [15].

In general, fragrance allergy affects at least $1 \%$ of the adult population [16]. According to the North American Contact Dermatitis Group (NACDG Patch Test Results 1998-2000), fragrances are the most common cosmetic allergen and the second most common allergen overall following metals [17]. Similar results were detected in our study as metals and fragrances were the most common positive patch test findings. However, our results are in contrast with those of Hillen et al. [4], who did not find fragrances to be a common cause of scalp contact dermatitis.

The possibility of scalp exposure to fragrances is quite high given the prevalence of fragrances in many products such as shampoos, conditioners, and hair tonics. A study examining products sold in the national retailer Walgreens found fragrances in $95 \%$ of shampoos [18]. A study that tested patients' own products found that hair cleansing products including shampoos were the third leading cause of contact dermatitis of the scalp [4].

Patch testing for both fragrance mix and balsam of Peru has been reported to identify $90 \%$ of all cases of fragrance allergy [13], with testing for balsam of Peru alone identifying $50 \%$ of cases of fragrance allergy. Therefore, testing both fragrance mix and balsam of Peru is advisable to identify a fragrance allergy.

\section{Carba Mix}

Carba mix was the third most common allergen in our patients after metals and fragrances, with a positive reaction recorded in 21 patients (11.6\%). Carba mix serves as a rubber accelerator added to natural rubber to speed its polymerization. It is used in the manufacture of many rubber products and is found in products made with natural rubber, butyl rubber, nitrile, or neoprene [14]. Examples of such products include office products (rubber bands, erasers, adhesives), industrial and safety products (ear plugs, goggles, headphones, masks, respirators, aprons), health care equipment (tubing, medical and utility gloves, bed sheeting, dental dams), and sports equipment (swim wear, wetsuits, elastics, shoes, boots, and racquet and club handles). In addition to rubber products, carba mix may also be found in fungicides, pesticides, antirust products, and in some soaps, shampoos, and disinfectants. Furthermore, rubber hairbrushes are very commonly utilized (particularly for detangling), and although not reported in the literature, can be a possible cause of scalp contact dermatitis.

Hair glues containing natural rubber latex are used by hairdressers to apply hair weaves and extensions. The latex in these adhesives has been reported to cause severe allergic reactions of the scalp, leading to anaphylaxis with symptoms of urticaria, angioedema, airway compromise leading to endotracheal intubation, and even death [1922]. This is an especially dangerous situation as the adhesives can be very difficult to remove from the scalp, resulting in hours of exposure to the allergen. Repeated exposure to hair glues may potentially sensitize consumers who frequently weave their hair [20]. Latex allergy should be considered when faced with a patient suffering from acute anaphylaxis with a history of a recent hair weave or extension placement, which is commonly found in certain ethnic groups such as African Americans [22, 23].

\section{Propylene Glycol}

PG was positive in 16 patients, which accounts for $8.8 \%$ of patients with a positive patch test result. These particular patients confirmed the use of scalp products containing PG, including topical minoxidil solution. PG is a main ingredient of topical minoxidil solution, representing a $20 \%$ component of the $2 \%$ solution and a $50 \%$ component of the $5 \%$ solution. PG is not present in minoxidil foam and is therefore a safer alternative for patients allergic to PG. Other hair products that might contain PG include shampoo, conditioner, hair gel, and topical steroids. PG was found in $40 \%$ of shampoos sampled in Walgreens shopping centers in North America [18]. PG is a common vehicle of topical drugs and cosmetic products. In a study by the NACDG (1996-2006) [24], personal care products were the most common source of
Allergens Identified in Patients with

Suspected Contact Dermatitis of the Scalp
Skin Appendage Disord 2017;3:7-14 DOI: $10.1159 / 000453530$ 
PG (54\%), followed by topical steroids (18\%) and other topical medications (10\%). PG is a strong irritant that can cause irritant patch test reactions. When used in products, it is difficult to include a concentration of PG that does not cause this irritation or allergic reaction [25].

Previous studies on scalp contact dermatitis have not identified PG as a common allergen [4]. This discrepant finding may be due to differences in our study population as we also run a hair clinic, and a significant proportion of our patients apply topical minoxidil or topical corticosteroids to treat various hair conditions.

\section{Ammonium Persulfate}

APS is a strong oxidizing salt used in the process of hair bleaching to decrease the quantity of hydrogen peroxide needed in the hair bleaching process [26]. APS was found to be positive in 13 patients (7.2\%) in our study population. This prevalence is slightly higher than in the study conducted by Hillen et al. [4], which found 3.2\% of subjects to have contact scalp dermatitis caused by APS. APS is also a frequent occupational allergen in hairdressers [27-30].

\section{Preservatives}

Positive patch tests to preservatives were found in 24 patients (13.3\%). Preservatives are commonly found in hair products such as shampoos, conditioners, hairstyling products, and hair dyes to prevent contamination and spoilage with bacteria, fungi, yeast, and algae. Preservatives are widely used in many personal, household, and industrial products. Positive patch test results included methylchloroisothiazolinone/methylisothiazolinones (Kathon CG), methyldibromoglutaronitrile (MDBGN), and quaternium-15. MDBGN and Kathon CG were both identified as allergens in $5.0 \%$ of our patients. A positive test for methylisothiazolinone was found in 2 patients. Our results differ slightly from those of Hillen et al. [4], who detected positive reactions to Kathon CG and MDBGN in 1.9 and $3.0 \%$ of their patients, respectively.

\section{Paraphenylenediamine}

PPD was positive in 6 patients (3.3\%). PPD is an arylamine derivative most frequently used in permanent hair dyes and also found in henna tattoos [31]. PPD is a very strong sensitizer, which makes it an essential screening test for scalp dermatitis [32]. A review study found the prevalence of PPD contact allergy to be $4.4 \%$ in Asia, $4.0 \%$ in Europe, and 6.0\% in North America [33].

PPD allergy was slightly less common in our population $(3.3 \%)$ when compared to the findings of the review study by Thyssen and White [33]. One explanation for this finding could be that we are a referral center and most cases of PPD allergy are not referred because PPD allergy is generally evident, making it a relatively easy condition to diagnose. Along with other hair coloring agents, PPD was among the most common allergens found to cause scalp contact dermatitis in the study performed by Hillen et al. [4].

\section{Minoxidil}

Minoxidil was tested in 73 patients in total, of whom 4 (5.5\%) had positive patch test results. Minoxidil frequently causes irritation, itching, and dryness of the scalp. These symptoms could be due to an irritant or to allergic contact dermatitis [34]. Patch testing is necessary in all patients complaining of scalp itching when utilizing topical minoxidil to determine if the patient is allergic to minoxidil itself (which is rare) or to PG (a common finding) [34]. PG is a component of minoxidil solution, but is not found in minoxidil foam products.

Contact dermatitis to minoxidil may look very similar to scalp psoriasis and may cause acute telogen effluvium in patients [35]. Persistent allergy to minoxidil can manifest as cutaneous lymphoid hyperplasia [36]. Photoallergic contact dermatitis in association with minoxidil is very rare [37]. In addition, pustular allergic contact dermatitis is an exceptional presentation [38]. Furthermore, the contact dermatitis caused by minoxidil can be occupational and affect the hands [39]. Videodermoscopy does not detect dermoscopic features to help distinguish scalp contact dermatitis due to topical minoxidil from other conditions that cause scalp itching, as no significant differences in vascular patterns or scaling have been found [40].

\section{Disperse Blue Dye}

Disperse blue dye tested positive in 7 patients (3.9\%). Disperse dyes are commonly used as textile coloring agents in dress clothing and are the most common fabric coloring agents to cause allergy. These dyes are also found in hair dye products and may be found in head bands, baseball cap bills, and synthetic wigs. Allergic reactions to disperse blue dye may be misinterpreted as PPD allergy due to cross-sensitivity between disperse blue dye and PPD, which is also found in hair dyes. However, studies have reported allergic contact dermatitis to disperse dyes without cross-sensitivity to PPD, including a recent study which found that $30 \%$ of patients allergic to disperse dyes would have gone undetected had PPD alone been tested $[41,42]$.
Aleid/Fertig/Maddy/Tosti 


\section{Limitations}

This study was a retrospective analysis. A relatively small number of patients were included in the study. Furthermore, follow-up data are lacking that would demonstrate that avoidance of specific allergens identified on patch testing led to improvement of the patient's symptoms after such offending agents were discontinued. We know from our anecdotal clinical experience that scalp dermatitis symptoms improved in the great majority of patients who avoided products that they were allergic to based on patch testing, but we do not have these followup data to track improvement of symptoms for every patient in this study. In addition, the selection process of our study may have resulted in a sample population that is not representative of the general population, since most patients were referred from a hair clinic.

\section{Conclusion}

Our study aggregates the data of patients afflicted by contact scalp dermatitis. This gives us a more comprehensive clinical perspective of the causes of contact dermatitis in patients referred for patch testing. Metals such as nickel and cobalt, along with fragrances, carba mix, and PG, were the most common allergens associated with contact scalp dermatitis identified in this study. Frequent sources of allergens included hair clasps, pins, and brushes for metals, while shampoos, conditioners, and hair gels were relevant for exposure to PG, fragrance mix, and balsam of Peru. Patients reported scalp itching or burning as the most common symptom. A scalp rash or itching of the scalp was reported in 177 cases $(78.3 \%)$. Other areas of the body were also affected in some patients, with the face being the most frequently involved area apart from the scalp (16.4\%). The majority of patients were females aged 40-59 years who had associated preexisting hair disorders, with androgenetic alopecia being the most common primary scalp condition, followed by telogen effluvium, seborrheic dermatitis, and alopecia areata. Only patients with scalp signs or symptoms that suggested a possible contact dermatitis were referred for patch testing, as this is not a common procedure for patients complaining of hair loss. However, it is important for clinicians to consider that allergic contact dermatitis of the scalp may cause hair loss and that patch tests might be very useful in a certain subset of patients [2].

In comparison with the study performed by Hillen et al. [4], similarities were found in positive patch test frequencies to nickel. However, some differences were observed as allergies to cobalt, fragrance mix, balsam of Peru, APS, and

preservatives (such as Kathon CG and MDBGN) were more frequent in our study, while allergies to PPD were less frequent. These differences may be attributed to the main focus on hair dye as the most common cause of scalp contact dermatitis in the Hillen et al. study, while hair dye was a less relevant allergen source in our patient population.

There are no epidemiological data concerning the prevalence of scalp contact dermatitis. However, while scalp contact dermatitis may not be very common, such a diagnosis should be suspected in patients with scalp itching, burning, or hair loss, especially if the patient has a recent history of utilizing a new medication or undergoing a hair treatment of any kind. The results of our study can inform clinicians about which allergens may be causing scalp contact dermatitis in patients and may serve to guide clinicians to patch test for certain allergens. Clinicians can also utilize these findings to inform patients about possible offending agents that may be the source of their scalp dermatitis based on the patient's individual patch test results.

\section{Statement of Ethics}

This study was approved by the University of Miami Institutional Review Board.

\section{Disclosure Statement}

Dr. Antonella Tosti received honoraria as a consultant, advisory board participant, speaker, or book author from the following companies: Aclaris, Incyte (consultant and principal investigator), Kythera, P\&G, DS Laboratories, Merck (consultant and speaker over 3 years ago), Taylor and Francis (author), Springer \& Verlag (author), and National Alopecia Areata Foundation (scientific board member). Raymond Fertig, Nouf M. Aleid, and Austin Maddy have no conflicts of interest to report.

\section{References}

Skin Appendage Disord 2017;3:7-14 DOI: $10.1159 / 000453530$
1 Huynh M, Sheehan MP, Chung M, Zirwas M, Feldman SR: Scalp; in Lewallen R, Clark A, Feldman SR (eds): Clinical Handbook of Contact Dermatitis. Diagnosis and Management by Body Region. Boca Raton, CRC Press, 2014, pp 6-12.

2 Tosti A, Piraccini BM, van Neste DJ: Telogen effluvium after allergic contact dermatitis of the scalp. Arch Dermatol 2001;137:187-190.

3 Pongpairoj K, Ale I, Andersen KE, Bruze M, Diepgen TL, Elsner PU, Goh CL, Goossens A, Jerajani H, Lachapelle JM, Lee JY, Maibach HI, Matsunaga K, Nixon R, Puangpet P, Sasseville D, Thaiwat S, McFadden JP: Proposed ICDRG classification of the clinical presentation of contact allergy. Dermatitis 2016;27:248-258.
Allergens Identified in Patients with

Suspected Contact Dermatitis of the Scalp 
4 Hillen U, Grabbe S, Uter W: Patch test results in patients with scalp dermatitis: analysis of data of the Information Network of Departments of Dermatology. Contact Dermatitis 2007;56:87-93.

5 Zug KA, Warshaw EM, Fowler JF Jr, Maibach HI, Belsito DL, Pratt MD, Sasseville D, Storrs FJ, Taylor JS, Mathias CG, DeLeo VA, Rietschel RL, Marks J: Patch-test results of the North American Contact Dermatitis Group 2005-2006. Dermatitis 2009;20:149-160.

6 Thyssen JP, Jensen P, Johansen JD, Menné T: Contact dermatitis caused by nickel release from hair clasps purchased in a country covered by the EU Nickel Directive. Contact Dermatitis 2009;60:180-181.

7 Starace M, Militello G, Pazzaglia M, Vincenzi C, Tosti A: Allergic contact dermatitis to nickel in a hair clasp. Contact Dermatitis 2007;56: 290.

8 Thyssen JP, Menné T, Johansen JD: Nickel release from inexpensive jewelry and hair clasps purchased in an EU country - are consumers sufficiently protected from nickel exposure? Sci Total Environ 2009;407:5315-5318.

9 Ono H, Takasuna H, Tanaka Y: Alopecia due to an allergic reaction to metal head-pins used in a neurosurgical operation. Surg Neurol Int 2016;7(suppl 1):S5-S7.

10 Sommer S, Wilkinson SM: Allergic contact dermatitis caused by a nickel-containing headband. Contact Dermatitis 2001;44:178.

11 Marks JG, Elsner P, DeLeo VA: Standard allergens; in: Contact and Occupational Dermatology, ed 3. Philadelphia, Mosby, 2002, pp 65-69.

12 Ruff CA, Belsito DV: The impact of various patient factors on contact allergy to nickel, cobalt, and chromate. J Am Acad Dermatol 2006;55:32-39.

13 Rystedt I, Fischer T: Relationship between nickel and cobalt sensitization in hard metal workers. Contact Dermatitis 1983;9:195-200.

14 Rietschel RL, Fowler J: Fisher's Contact Dermatitis. PMPH-USA, 2008, p 885.

15 Salam TN, Fowler JF: Balsam-related systemic contact dermatitis. J Am Acad Dermatol 2001;45:377-381.

16 Frosch PJ, Pilz B, Andersen KE, Burrows D, Camarasa JG, Dooms-Goossens A, Ducombs G, Fuchs T, Hannuksela M, Lachapelle JM, et al: Patch testing with fragrances: results of a multicenter study of the European Environmental and Contact Dermatitis Research Group with 48 frequently used constituents of perfumes. Contact Dermatitis 1995;33:333342.

17 Marks JG Jr, Belsito DV, DeLeo VA, Fowler JF Jr, Fransway AF, Maibach HI, Mathias CG, Pratt MD, Rietschel RL, Sherertz EF, Storrs FJ, Taylor JS; North American Contact Dermati- tis Group: North American Contact Dermatitis Group patch-test results, 1998 to 2000. Am J Contact Dermat 2003;14:59-62.

18 Zirwas M, Moennich J: Shampoos. Dermatitis 2009;20:106-110.

19 Pumphrey RS, Duddridge M, Norton J: Fatal latex allergy. J Allergy Clin Immunol 2001; 107:558.

20 Cogen FC, Beezhold DH: Hair glue anaphylaxis: a hidden latex allergy. Ann Allergy Asthma Immunol 2002;88:61-63.

21 Wakelin SH: Contact anaphylaxis from natural rubber latex used as an adhesive for hair extensions. Br J Dermatol 2002;146:340-341.

22 Burla MJ, Brody AM, Welch RD, Favot MJ: Anaphylactic reaction after ongoing exposure to hair glue: a novel case report. J Emerg Med 2015;48:e5-e7.

23 McMichael AJ: Ethnic hair update: past and present. J Am Acad Dermatol 2003;48(suppl 6):S127-S133.

24 Warshaw EM, Botto NC, Maibach HI, Fowler JF Jr, Rietschel RL, Zug KA, Belsito DV, Taylor JS, DeLeo VA, Pratt MD, Sasseville D, Storrs FJ, Marks JG Jr, Mathias CG: Positive patch-test reactions to propylene glycol: a retrospective cross-sectional analysis from the North American Contact Dermatitis Group, 1996 to 2006. Dermatitis 2009;20:14-20.

25 Lessmann H, Schnuch A, Geier J, Uter W: Skin-sensitizing and irritant properties of propylene glycol. Contact Dermatitis 2005; 53:247-259.

26 Fisher AA, Dooms-Goossens A: Persulfate hair bleach reactions. Cutaneous and respiratory manifestations. Arch Dermatol 1976; 112:1407-1409.

27 Uter W, Gefeller O, John SM, Schnuch A, Geier J: Contact allergy to ingredients of hair cosmetics - a comparison of female hairdressers and clients based on IVDK 2007-2012 data. Contact Dermatitis 2014;71:13-20.

28 Lyons G, Roberts H, Palmer A, Matheson M, Nixon R: Hairdressers presenting to an occupational dermatology clinic in Melbourne, Australia. Contact Dermatitis 2013;68:300306.

29 Guerra L, Tosti A, Bardazzi F, Pigatto P, Lisi P, Santucci B, Valsecchi R, Schena D, Angelini G, Sertoli A, et al: Contact dermatitis in hairdressers: the Italian experience. Gruppo Italiano Ricerca Dermatiti da Contatto e Ambientali. Contact Dermatitis 1992;26:101107.

30 Schwensen JF, Johansen JD, Veien NK, Funding AT, Avnstorp C, Osterballe M, Andersen KE, Paulsen E, Mortz CG, Sommerlund M, Danielsen A, Andersen BL, Thormann J, Kristensen O, Kristensen B, Vissing S, Nielsen $\mathrm{NH}$, Thyssen JP, Søsted H: Occupational contact dermatitis in hairdressers: an analysis of patch test data from the Danish Contact Dermatitis Group, 2002-2011. Contact Dermatitis 2014;70:233-237.

31 Xie Z, Hayakawa R, Sugiura M, Kojima H, Konishi H, Ichihara G, Takeuchi Y: Experimental study on skin sensitization potencies and cross-reactivities of hair-dye-related chemicals in guinea pigs. Contact Dermatitis 2000;42:270-275.

32 Koopmans AK, Bruynzeel DP: Is PPD a useful screening agent? Contact Dermatitis 2003;48: 89-92.

33 Thyssen JP, White JML: Epidemiological data on consumer allergy to p-phenylenediamine. Contact Dermatitis 2008:59:327-343.

34 Friedman ES, Friedman PM, Cohen DE, Washenik K: Allergic contact dermatitis to topical minoxidil solution: etiology and treatment. J Am Acad Dermatol 2002;46:309-312.

35 La Placa M, Balestri R, Bardazzi F, Vincenzi C: Scalp psoriasiform contact dermatitis with acute telogen effluvium due to topical minoxidil treatment. Skin Appendage Disord 2015;1:141-143.

36 García-Rodiño S, Espasandín-Arias M, Suárez-Peñaranda JM, Rodríguez-Granados MT, Vázquez-Veiga $\mathrm{H}$, Fernández-Redondo $\mathrm{V}$ : Persisting allergic patch test reaction to minoxidil manifested as cutaneous lymphoid hyperplasia. Contact Dermatitis 2015;72: 413-416.

37 Tosti A, Bardazzi F, De Padova MP, Caponeri GM, Melino M, Veronesi S: Contact dermatitis to minoxidil. Contact Dermatitis 1985; 13:275-276.

38 Rodríguez-Martín M, Sáez-Rodríguez M, Carnerero-Rodríguez A, Cabrera de Paz R, Sidro-Sarto M, Pérez-Robayna N, Guimerá F, Sánchez R, García-Bustínduy M, Noda-Cabrera A: Pustular allergic contact dermatitis from topical minoxidil 5\%. J Eur Acad Dermatol Venereol 2007;21:701-702.

39 Veraldi S, Benelli C, Pigatto PD: Occupational allergic contact dermatitis from minoxidil. Contact Dermatitis 1992;26:211-212.

40 Tosti A, Donati A, Vincenzi C, Fabbrocini G: Videodermoscopy does not enhance diagnosis of scalp contact dermatitis due to topical minoxidil. Int J Trichology 2009;1:134-137.

41 Søsted H, Nielsen NH, Menné T: Allergic contact dermatitis to the hair dye 6-methoxy-2-methylamino-3-aminopyridine $\mathrm{HCl}$ (INCI HC Blue no. 7) without crosssensitivity to PPD. Contact Dermatitis 2009; 60:236-237.

42 Isaksson M, Ale I, Andersen KE, Diepgen T, Goh CL, Goossens RA, Jerajani H, Maibach HI, Sasseville D, Bruze M: Patch testing to a textile dye mix by the International Contact Dermatitis Research Group. Dermatitis 2015; 26:170-176. 